\title{
La radio generalista en la red: un nuevo modelo para la radio tradicional ${ }^{*}$
}

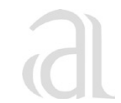

\author{
María del Pilar Martínez Costa \\ Elsa Moreno \\ Avelino Amoedo
}

Recibido: 20 de febrero de 2012 Aprobado: 27 de marzo de 2012

\begin{abstract}
Resumen
La radio generalista enfrenta un cambio sustancial en la denominada sociedad de la comunicación interactiva. Internet -como catalizador del proceso de convergencia digital-plantea un nuevo entorno comunicativo para la radio, que exige repensar las estrategias, los conceptos y la dinámica tradicional con la que viene trabajando el medio radiofónico. La radio generalista en la red adquiere una dimensión informativa novedosa que implica un modelo narrativo, productivo y programático original para atender la escucha en directo y bajo demanda, así como para fomentar la interactividad y el diálogo con el oyente. Este artículo de reflexión tiene como objeto perfilar el nuevo estatus de la radio generalista en internet.
\end{abstract}

Palabras clave: radio generalista, radio en la red.

\footnotetext{
Este trabajo se ha desarrollado en el marco del Subproyecto MICINN 2009/11 Evolución de los cibermedios españoles en el marco de la convergencia. Multiplataforma e integración periodística financiado por el Ministerio de Ciencia y Tecnología del Gobierno de España (CSO2009-13713-C05-03)

"* Doctora en Ciencias de la Información de la Universidad de Navarra, España. Actualmente se desempeña como Profesora Agregada y Vicedecana de Alumnos de Facultad de Comunicación de la Universidad de Navarra. Desde mediados de los años noventa investiga en la introducción de los sistemas de radio digital en Europa y los cambios que la tecnología digital ha desencadenado en la forma de pensar, hacer y gestionar los contenidos de la radio. Autora del libro La radio en la era digital (El País Aguilar, Madrid, 1997) pionero en esta materia; Reinventar la radio (Eunate, Pamplona 2001), Información radiofónica (Ariel, Barcelona 2002), Programación radiofónica (Ariel, Barcelona, 2004), Lenguaje, géneros y programas de radio (Eunsa, Pamplona, 2005), La crónica radiofónica (IORTV, Madrid, 2008).Correo electrónico: marcosta@unav.es

*.* Doctora en Comunicación por la Universidad de Navarra (1998). Profesora Adjunta de Programación Radiofónica, del Departamento de Proyectos Periodísticos, de la Facultad de Comunicación de la Universidad de Navarra. Investiga el diseño, la producción, la promoción y la comercialización de productos radiofónicos en el entorno digital, así como la evolución de los conceptos de radio, las estrategias empresariales y narrativas (criterios y técnicas). Autora de varios estudios sobre los modelos de programación radiofónica, entre ellos, la radio generalista y la radio musical. Correo electrónico: emoreno@unav.es

*.** Doctor en Comunicación por la Universidad de Navarra, España. Profesor Adjunto de Producción Radiofónica y Secretario del Departamento de Proyectos Periodísticos de la Facultad de Comunicación de la Universidad de Navarra. Autor de numerosos estudios sobre la estructura de la radio en el País Vasco. Su investigación se centra en la producción radiofónica: aspectos informativos, técnicos y en la estructura de la radio en España (historia, legislación, propiedad y programación).Correo electrónico: avamoedo@unav.es
} 


\title{
Generalist Radio on the Web: A New Model for Traditional Radio
}

\begin{abstract}
Generalist radio is facing a meaningful change within the renamed interactive communication society. The Internet, as a catalyzer of the digital convergence process, provides a new communicational environment for the radio, which demands rethinking strategies, concepts, and traditional dynamic radio media are working with. Generalist radio on the web acquires an innovative information dimension which implies an original narrative, productive, and programmatic model for supplying listening in a direct way and under demand, as well as for promoting interactivity and dialogue with listeners. This reflection article is intended to show the new status of generalist radio on the Internet.
\end{abstract}

Key words: Generalist radio; radio on the web. 


\section{Introducción: La radio generalista y la red}

Como en sus orígenes, la radio en la actualidad apela a la audiencia a través de diferentes opciones de emisión y distribución (Albarran $\mathcal{E}$ Pitts, 2001, p. 172). De todos los soportes digitales, Internet se presenta como el espacio impulsor de la nueva radio que exige la actualización de las estrategias, los conceptos y hábitos de trabajo con los que viene trabajando el medio tradicional para atender la relación con los ciudadanos de la denominada sociedad de la e-comunicación -digital, convergente y globalizadora-.

El desarrollo de Internet plantea un nuevo entorno comunicativo para la radio. Una nueva forma de radio según algunos autores (Wall, 2004, p. 29; Priestman, 2004, p. 77), que otorga una dimensión singular a la relación de siempre entre el oyente -actual usuario-, y los profesionales y contenidos de la marca radiofónica (Priestman, 2002, p. 228). Puede decirse que la naturaleza multimedia e interactiva de la red amplía el espacio comunicativo entre una cadena de radio y su público. Además, Internet se presenta como una ocasión para enriquecer las estrategias discursivas del medio radiofónico, desarrollar contenidos más personalizados e intensificar el diálogo con el grupo de oyentes que se identifican con un estilo particular de "hacer radio". Finalmente, el sitio web es una herramienta más para promover los contenidos de la antena y para dotar de un nuevo valor a la distribución de la programación basada en la interactividad (Pitt E Daños, 2003, p. 271).

En el caso particular de la radio generalista, la continuidad informativa de la antena tradicional -elemento esencial de esta estrategia radiofónicapuede incrementarse con las noticias y el análisis de la actualidad, ofrecido al oyente en el sitio web de una cadena. Es decir, a través de la estrategia informativa ideada y realizada en Internet, máxime cuando este medio avanza en penetración social y tiende a convertirse en un referente claro de la actualidad general y especializada en la mayoría de las sociedades y mercados. Asimismo, el conjunto de contenidos que conforman la oferta específica de cada uno de los programas magacines y los programas temáticos de este tipo de radio se extienden también hacia la red con un desarrollo propio.

La radio generalista española muestra un interés cada vez mayor hacia la red impulsando los equipos de profesionales y las estructuras de producción del contenido radiofónico para este canal, y mejorando su presentación y diseño para favorecer la interactividad y el uso de los recursos multimedia'. Sin embargo, las cadenas generalistas españolas disponen de actitudes distintas en sus páginas, especialmente las referidas al trabajo periodístico. Incluso, en algunos casos, la presencia informativa se crea sobre una base muy limitada.

Las investigaciones de carácter teórico y práctico que analizan la relación radio y red coinciden en afirmar que la radio en Internet presenta un desafío en concepto y términos de definición, como es el caso de Coyle (2006, p. 123). En este sentido, son numerosos los autores que han destacado la importancia de redefinir tácticas, productos y contenidos que atiendan a las demandas del nuevo modelo de radio en la convergencia digital ${ }^{2}$.

Internet impone repensar y replantear la radio (Cebrián Herreros, 2008, p. 133): "nace otro producto. A medida que se profundiza y se experimenta surgen otras modalidades sonoras que

1 La totalidad de las cadenas radiofónicas españolas de ámbito nacional han renovado sus sitios web a lo largo de las últimas cinco temporadas, con el propósito principal de fomentar la interactividad con el oyente (Moreno, Amoedo \& Martínez-Costa, 2008). Esta tendencia también se percibe en otros mercados como el norteamericano (Seelig, 2008).

2 Ala-Fossi, Lax, O'Neill, Jauert \& Shaw (2008); Cebrián Herreros (2008); Berry (2006); Evans (2005); Priestman (2004 y 2002); Ren E Chan-Olmsted (2004); Wall (2004); Hamula \& Wenmouth (2003); Moody, Greer \& Linn (2003); Pitts \& Harms (2003); Ribes i Guàrdia (2003); Randle E Mordock (2002); Hendy (2000); Tacchi (2000); Evans \& Smethers (2001); Lind \& Medoff (1999), entre otros. 
amplían o modifican la concepción tradicional del medio". El medio radiofónico está redefiniéndose y reinventándose (Faus, 2001, p. 16). Asiste a otra radio-mutación o cambio radiofónico, entendido este término en el sentido propuesto por Calderón (1972, p. 21): "las evoluciones observadas en la forma de contar como consecuencia de un modo de entender lo radiofónico, a lo largo de su evolución en el tiempo".

De ahí que, la implantación de la tecnología digital haya reavivado el interés por los estudios y la investigación en radio (Tacchi, 2000, p. 289). Especialmente en este momento, en el que el futuro de la radio es mucho menos obvio y claro que las previsiones de hace diez años (Ala-Fossi, Lax, O'Neill, Jauert E Shaw, 2008, p. 4) . $^{3}$

Todas estas cuestiones de fondo y de forma que plantea la consideración de las características esenciales de la comunicación radiofónica en Internet se perciben como relevantes en la línea de investigación iniciada por los autores hace 5 años y que aborda el estudio comparativo de las estrategias de comunicación de la radio española en la red ${ }^{4}$ desde una perspectiva práctica. La conclusiones obtenidas tras el análisis particular de las páginas web de las cadenas generalistas SER, COPE, Onda Cero, Punto Radio y Radio Nacional permite aproximarse a un replanteamiento de la pauta tradicional con la que viene trabajando esta oferta desde la antena y cuya aplicación en la red debe aprovechar la naturaleza comunicativa propia de este medio. Un asunto subrayado por la bibliografía académica desde una perspectiva más teórica y que este trabajo también tiene en cuenta ${ }^{5}$.

3 Radio in the future was expected to offer much more personalized and specialized content, also with multimedia elements, which would then be actively selected by the users for listening whenever they found it suitable (Ala-Fossi, Lax, O'Neill, Jauert E Shaw, 2008: 17).

4 Véase al respecto otros estudios presentados y publicados por los autores en la bibliografía.

5 La hipertextualidad, la multimedialidad-integración del texto, la
El objeto de este artículo es describir el nuevo estatus de la radio generalista, que adquiere una magnitud informativa novedosa al compatibilizar el relato de las noticias en la antena y en Internet. Asimismo, este tipo de radio -creada en torno a la actualidad del día, el entretenimiento y el contenido local-, y esta estrategia empresarial -dirigida a la totalidad de la audiencia variada- enfrentan otra forma de entender, realizar y proponer el producto radiofónico al oyente a través de la red. Es necesario atender a una escucha que, por un lado se torna más personal y segmentada, y por otro, se integra en el "compartir" del fenómeno de las redes sociales.

\section{Las estrategias de la radio generalista española en la red}

El objetivo de la investigación realizada entre 2006/2007 y 2010/2011 ha consistido en evaluar la presencia de las cadenas SER, COPE, Onda Cero, Punto Radio y Radio Nacional en Internet, así como profundizar en el análisis cuantitativo y cualitativo de la estrategia informativa de estas páginas web. Se ha determinado si las cadenas generalistas españolas de ámbito nacional proponen al oyente una oferta original de contenidos, aprovechan las posibilidades multimedia de la red y diversifican las técnicas de interactividad, especialmente, respecto del contenido informativo.

El estudio se ha elaborado parcial y progresivamente en distintos períodos de tiempo en cada temporada. Para ello, se ha realizado un seguimiento periódico de las páginas web de las citadas cadenas radiofónicas. Las muestras se han tomado en dos semanas distintas correspondientes a cada temporada de programación desde el curso 2006/2007 hasta la el período 2010/2011. Para realizar el trabajo de campo se ha empleado

imagen, el vídeo y el sonido para crear una unidad comunicativa coherente- y la interactividad, además de la simultaneidad o actualización de las noticias en la red son las características que definen al lenguaje ciberperiodístico, según Salaverría (2005). 
una ficha de análisis de contenido que recoge la evolución de cuatro elementos de estudio:

1. Las temáticas de la antena tradicional que se destacan en la página principal del sitio web de cada cadena radiofónica.

2. Los contenidos complementarios que, siendo propios de la antena, adquieren un valor añadido en la red por su tratamiento y disponibilidad de acceso.

3. Los servicios asociados que se presentan de forma exclusiva en la página web de cada cadena.

4. Las técnicas de interactividad principales que fomentan la participación del oyente.

Al mismo tiempo, esta observación se ha ampliado en algunas semanas concretas con la captura sistemática de muestras de las páginas web de las cadenas radiofónicas para realizar un análisis comparativo del contenido de actualidad publicado entre las 12 y las 14 horas de los días convenidos para el trabajo de campo. Los autores han capturado diversas muestras de las páginas web de las cadenas para hacer un análisis comparativo, siguiendo el criterio temporal de emisión horaria de los programas informativos: los boletines de las 12 y las 13 horas, y el programa informativo principal del mediodía a las 14 horas ("Hora 14" en la Cadena SER-, "La palestra" en la COPE, "Noticias mediodía" en Onda Cero, "Primera plana" en Punto Radio y "14 Horas" en Radio Nacional). Se ha seleccionado este tramo de programación porque se corresponde con el momento informativo de transición y evaluación de la actualidad que se genera durante la mañana y cuyo contenido produce y emite la radio generalista española.

A cada una de las muestras se ha aplicado una ficha de análisis de contenido según los siguientes cuatro elementos de estudio:

1. El número de noticias que cada cadena ofrece al oyente en la página principal de su web.
2. El tipo de noticias o los temas informativos con los que se corresponden.

3. El tratamiento multimedia y narrativo de las tres noticias principales publicadas en la portada de la web de la cadena: las fuentes utilizadas, el número de palabras empleadas en la configuración del titular y del texto, si se trabaja con sonidos, fotos, vídeos, otros elementos gráficos, enlaces o documentos asociados.

4. Las técnicas interactivas, propias de la red, disponibles para el usuario, como son la posibilidad de insertar comentarios en la noticia y compartirla, de manifestar interés personal hacia el contenido informativo o de consultar su popularidad entre la comunidad de oyentes de la cadena.

Asimismo, y para constatar si existe una sinergia de la estrategia periodística de la radio generalista española en la red y en la antena tradicional, este trabajo también ha considerado el contenido de las noticias principales de los boletines horarios de las 12 y de las 13 horas, así como los temas de apertura de los programas informativos de mediodía. Es decir, también se han estimado las muestras sonoras de la antena. La ampliación del análisis a las temáticas de los programas informativos de este soporte durante el período horario analizado ha permitido identificar las cadenas que trabajan una táctica informativa coordinada para ambos canales.

A modo de conclusión general, se comprueba una mejora notable de las presencia en Internet de las cadenas generalistas -SER, COPE, Onda Cero, Punto Radio y Radio Nacional- entre 2006 y 2011 , especialmente del diseño, la navegabilidad y las opciones multimedia de algunas páginas web, de entre las que sobresalen los sitios de las cadenas SER, COPE y Radio Nacional.

La interactividad -aplicada al diálogo permanente con el oyente- es el recurso prioritario que 
más valoran los sitios web analizados, que tienen los desafíos de idear nuevos productos, ahondar en la diversificación temática -de acuerdo con el interés general y las preferencias específicas-, valorar el sonido radiofónico, y aprovechar la sindicación de servicios asociados de carácter noticioso y/o comercial. La radio española también puede encaminarse hacia la producción de contenidos generados por el oyente en el entorno de la Web 2.0.

Asimismo, y de manera particular con respecto del contenido de la actualidad, las cadenas SER, COPE, Onda Cero, Punto Radio y Radio Nacional de España tienen el desafío de mejorar la edición informativa complementaria para la red-Noticias, Informes Especiales y Coberturas en directo-, así como su actualización constante de acuerdo con el discurrir y seguimiento de cada noticia aprovechando las posibilidades narrativas de la red y la sinergia de los tiempos de producción y de emisión de la continuidad informativa de la antena. Este reto cobra relevancia en el mercado multimedia actual en el que otros medios tradicionales, como la prensa, trabajan ya con el concepto editorial de "cierre continuo" aproximándose al ritmo informativo de la radio generalista tradicional.

La hipertextualidad y la multimedialidad de la red amplían y complementan las posibilidades expresivas del sonido para relatar y analizar la actualidad general y especializada, desde criterios expositivos y narrativos más comprensibles y amenos. Sirva el ejemplo de que las noticias de información económica y financiera son las más consultadas por los usuarios de la Cadena SER.

También, la interactividad de Internet incrementa la capacidad de difundir, recibir y compartir información entre la radio y los ciudadanos -de manera individual o a través de redes sociales-. La radio generalista en Internet conviene que no olvide que las nuevas modalidades interactivas de participación radiofónica son también excelentes técnicas para conocer cualitativamente a las audiencias y así atender sus demandas informativas y de entretenimiento. En definitiva, aprovechar los recursos narrativos e interactivos de la red para fortalecer la comunicación radiofónica.

El trabajo de campo realizado sobre las habilidades de comunicación de la radio generalista española en Internet -con interés especial respecto del trabajo informativo- y la consideración de las ideas teóricas principales sobre la radio y la red ayudan a establecer los principales cambios que afronta este tipo de radio, la cual también participa de la reconfiguración del mercado informativo español en la convergencia digital.

\section{Hacia un nuevo concepto de radio}

A partir de este mapa de la radio generalista en la red, se constata que la transformación de los estándares radiofónicos en la red introduce modificaciones teóricas y prácticas en todos los ámbitos de trabajo que afectan la gestión de contenidos de la radio generalista, entre ellos, los informativos. En los siguientes epígrafes delimitaremos los aspectos que redefinen al medio en su adaptación a las características de los nuevos entornos creados a partir de la red, en cuatro ámbitos concretos: a) el modelo narrativo; b) el modelo de producción; c) el modelo de programación; d) el modelo de participación; y e) la marca radiofónica y su valor añadido.

\subsection{El modelo narrativo}

Internet comenzó ofreciendo la posibilidad de un nuevo soporte de emisión para luego proyectarse como un nuevo medio con una narrativa propia. Cuando la radio utiliza la red como soporte de distribución multiplica sus posibilidades de emisión en directo, y diferido, a audiencias totales y bajo demanda. En un primer momento, las emi soras se centraron en la distribución de la oferta del canal tradicional de radio para, en un segundo estadio, abrir posibilidades de nuevas formas de organización y combinación de contenidos, cons- 
tituyendo una nueva propuesta de presentación de la actualidad y el entretenimiento.

Pero limitarse a esta multiplicación de canales disponibles es una forma muy simplificada de entender la red porque ha terminado por imponer un nuevo lenguaje -multimedia- y una nueva forma de narrar -interactividad-. Con el lenguaje multimedia se abren posibilidades de ofrecer audio, texto, fotos, gráficos y vídeo, cada uno con sus respectivos códigos de funcionamiento, pero integrados en un único relato. Con la narrativa interactiva, por su parte, surge la posibilidad de aumentar, completar, actualizar, enlazar, navegar y compartir los contenidos generados por el emisor original, por otras fuentes o por el mismo usuario, el cual participa ahora de un proceso de producción de contenidos abierto a sus aportaciones.

En los estudios realizados hasta la fecha se comprueba que en 2006 la radio generalista en España aprovechaba solo la capacidad de ofrecer texto escrito en el nuevo soporte, para luego aumentar la disponibilidad del sonido de la antena a demanda y, finalmente, producir material audiovisual propio o asociarlo a la oferta del grupo de comunicación. Sin embargo, son aún escasas y excepcionales las coberturas en las que la radio generalista trabaja sobre una concepción realmente multimedia e interactiva para sus sitios en Internet ${ }^{6}$.

Con respecto a la narrativa interactiva, y aunque se desarrollarán otros aspectos más adelante, tampoco hay un total aprovechamiento de las posibilidades de enlazar, navegar y compartir contenidos informativos. La producción de materiales por parte del usuario se ha limitado a la

6 Queda pendiente realizar también un estudio exhaustivo de las características de los textos y su adaptación a la red. Aunque se observa que progresivamente se presentan titulares más cortos y directos, aún predomina en la redacción el estilo tradicional de los textos periodísticos y no se asumen como propios los parámetros de redacción para web. Véase al respecto, por ejemplo: Salaverría (2005), Canavilhas (2007) y Franco (2008), entre otros. realización de encuestas asociadas a los temas de portada y, ocasionalmente, a la aportación de material gráfico. En abril de 2008 y en pleno auge del periodismo ciudadano, la Cadena SER comenzó a desarrollar un espacio informativo para la elaboración de los usuarios bajo la denominación "SER Periodista", en donde los oyentes aportan historias, generalmente locales, aunque no suelen integrarse al discurso informativo de la página principal ni a la antena.

Tampoco está resuelta aún la sinergia y retroalimentación entre los contenidos y tratamientos narrativos de la antena y de la red. Es cierto que el seguimiento de la estrategia de las emisoras generalistas en la red se observa una mayor visibilidad y un mejor aprovechamiento de los productos sonoros y radiofónicos producidos para la antena: hay más sonido disponible en cada noticia y hay programas completos disponibles a demanda o suscripción. Pero no hay una buena catalogación y etiquetado de materiales de archivo, sean de audio, texto, vídeos o fotografías, ni hay herramientas de búsquedas adecuadas que faciliten el acceso a esos contenidos. Aunque el estudio no ha sido exhaustivo en la antena, tampoco se produce una sinergia de contenidos de la red a programación de la antena, a excepción de las encuestas, entrevistas digitales y anuncios de la página web y de las posibilidades de participación que allí se ofrecen, fundamentalmente para los programas magacines, pero en menor medida para los programas informativos.

Por tanto, podemos afirmar que la radio generalista española en la red tiene aún que trabajar en el desarrollo de este nuevo lenguaje y de esta nueva narrativa, no sólo como añadidos o complementos que "ilustran" su contenido de audio. Tiene que entender que en la red los lenguajes se integran en una nueva forma de entender la comunicación, y por tanto, lo radiofónico en la red. Cada sistema de códigos -texto escrito, sonido, imágenes, gráficos- tiene que adaptarse a la red e integrarse de forma adecuada en el 
lenguaje multimedia y en la narrativa abierta de la interactividad.

Ahora bien, el sonido y lo radiofónico, y sus diversas formas de presentación son el valor diferencial que una cadena generalista puede ofrecer en la red. En este sentido, hay aún márgenes para un mejor aprovechamiento del sonido de la antena, sobre todo rentabilizando la capacidad de indexación, etiquetado y recuperación de archivos en la red.

\subsection{El modelo de producción}

En el inicio de nuestro estudio partíamos de los parámetros de ideación, producción y programación de los contenidos de la radio generalista tradicional. Los criterios de selección y valoración de noticias y temas de actualidad, los ritmos de producción y emisión, y la estructura y tratamiento narrativo tienen sus peculiaridades en la antena. Se caracterizan por el uso exclusivo del sonido, la narración siempre en presente, el estilo conversacional y la necesidad de construir y mantener el interés del oyente (Martínez-Costa 2002, pp. 95-100). Estas condiciones de partida tienen luego consecuencias sobre la redacción y la presentación ante el micrófono.

La radio generalista tradicional trabaja sobre la inmediatez, la simultaneidad y un ritmo de actualización constante de la información siguiendo el discurrir del acontecer diario, y esto se percibe en toda la programación. Precisamente, la inmediatez, la simultaneidad y la actualización son parte de las ventajas tradicionales de la radio, en tanto el medio aproxima el tiempo informativo al tiempo del relato, es decir, al tiempo de emisión y recepción. La continuidad informativa tradicional es lineal, se mantiene en tensión las 24 horas del día y se organiza en torno a la convención de la unidad narrativa de la hora. Hay sucesivas actualizaciones en los boletines horarios, tres momentos para la recapitulación o síntesis en los tiempos informativos de los magacines y los programas informativos -por la mañana de 6 a 9 horas, al mediodía de 14 a 15 horas y por la noche de 20 a 24 horas-. Asimismo, los programas magacín de mañana y tarde también abordan la actualidad del día desde diferentes perspectivas haciendo hincapié en el entretenimiento, la participación de la audiencia, el contenido social y el entorno más próximo al oyente.

Este ritmo de producción, de emisión y recepción de la antena tradicional es diferente en la red, donde uno de los condicionantes de trabajo o la unidad de medida no es la hora, ni el tiempo de emisión o recepción, sino la disponibilidad de espacio. El espacio está limitado por las dimensiones de la pantalla y de las posibilidades de navegación entre diferentes niveles o páginas. En Internet el tiempo no es tiempo de emisión, sino tiempo de acceso y navegación del usuario que repasa los contenidos de forma fragmentada y no lineal. Unos contenidos que también hay que actualizar de acuerdo con determinados tiempos de publicación.

También puede haber inmediatez y simultaneidad en la red, pero el análisis realizado de las páginas web de las cadenas generalistas españolas muestra que no predomina el criterio de la última hora en la renovación de las noticias, sino la facilidad de acceso o usabilidad y la jerarquización temática de contenidos. La urgencia marca la selección y valoración de noticias y temas en la antena. La proximidad temporal -algo acaba de ocurrir, está ocurriendo o va ocurrir en los próximos minutos- es determinante en el soporte tradicional. Por el contrario, los sitios web evaluados no atendían tanto la inmediatez informativa en las primeras muestras obtenidas, sino que realizaban una apuesta temática estratégica que se traducía en un número prácticamente estable de noticias a incluir en secciones fijas tanto de la página principal como de los programas. Desde la temporada radiofónica 2008/2009 y de forma más generalizada en la 2009/2010 se incluyen en la red secciones de "Última hora", "Noticias urgentes" o "Al minuto" con uno o dos titulares. 
Por otra parte, la producción y edición del discurso informativo aparecen claramente diferenciadas del relato y la presentación de los programas. La elaboración de las noticias ha adquirido un tratamiento diferenciado y propio para la red, mientras que para la exposición de los programas predomina el simple volcado de contenidos de la antena y se ha realizado una apuesta por desarrollar los diferentes sistemas de participación de la audiencia.

Por ello, se puede afirmar que en la antena prima la urgencia, la última hora, la actualización constante y continuidad del relato informativo en toda la programación, mientras que las cadenas generalistas españolas no aprovechan la posibilidad de renovación que permite la red. Respecto del contenido informativo, solo se percibe un número estable de noticias con una apuesta temática propia por cadena organizada de acuerdo con secciones distribuidas en un espacio que acumula progresivamente datos y tratamientos complementarios. Así, la producción de contenidos para la antena se caracteriza por la síntesis y la continuidad, mientras que en la red predomina el desarrollo complementario y la suma fragmentada de contenidos.

Hay que señalar también que, este nuevo modelo de producción que se impone en la red está modificando la organización del trabajo en las redacciones de las emisoras. La radio generalista comienza a organizar su sistema de trabajo en función de los contenidos y, en consecuencia, a abandonar la estructura de secciones heredada de la prensa escrita y diferenciadas en función de la tecnología y los soportes de difusión. Por esta razón, la planificación de coberturas especiales debe obedecer a decisiones estratégicas sobre los contenidos e incluir acciones que respondan a las características específicas de cada soporte, sin perder la identidad del medio, y encontrar las sinergias y retroalimentaciones posibles entre las diferentes plataformas.

\subsection{El modelo de programación}

Por lo expuesto hasta el momento, y gracias a la interactividad y capacidad de almacenaje de la red, puede decirse que la oferta radiofónica adquiere una perspectiva y significado nuevo en Internet. Este medio permite desarrollar una radio más personal e interactiva - "radio de cada oyente"-, que atienda tanto la escucha en directo -simulcasting de la antena en la red-, como la escucha bajo demanda -entendida esta consulta individual como la descarga de archivos y el servicio de suscripción de determinados contenidos-. El acceso diacrónico a la red facilita que cada usuario configure una navegación temática y audición personalizada de entre la propuesta de contenidos en directo y diferido que la cadena ofrezca al oyente en Internet. Esta opción de escucha personal se presenta como un valor añadido frente a la escucha convencional.

En el caso de la radio generalista, la oferta de un canal único de audio, lineal y cerrado en la antena basado en la variedad de contenidos y dirigido a la totalidad del público se amplía hacia la posibilidad de ofrecer una radio más individual, no lineal y abierta al usuario interesado en determinadas temáticas de la actualidad y el entretenimiento.

El estudio realizado comprueba que la radio generalista española tiene el desafío de ofrecer asuntos originales y de producción propia para la red. No obstante, sí se percibe una apuesta diferente de las modalidades de presentación y combinación de los contenidos de la antena en Internet. La mayoría del conjunto de audios, sonidos, piezas, secciones y programas que conforman las 24 horas de la programación tradicional ganan en autonomía, y "rompen" la continuidad y secuencialidad de la antena para proponerse e integrarse en formatos de exposición singulares en la página web. 
Puede decirse que las unidades narrativas de toda programación -también las informativasadquieren un valor discursivo diferente en Internet (Moreno, 2008, p. 385). Priestman destaca este aspecto al mencionar el protagonismo que adquiere, por ejemplo, el programa con respecto de la programación (2002. p. 218). Por lo tanto, la convergencia de la red y la antena implica que el producto radiofónico ya no puede ser concebido para un solo medio de emisión. Según Faus, la distribución alternativa debe ser tomada en consideración desde la fase de planificación de la producción de programas (2001, p. 37).

Así, la estructura de la parrilla tradicional -basada principalmente en la linealidad del directo, la organización horaria, la adecuación de la variedad del contenido a los tiempos sociales, radiofónicos y el nivel de audiencia de cada momento- evoluciona hacia diferentes arquetipos o esquemas de combinación de los contenidos informativos y de entretenimiento que se escuchan en directo, de los que se descargan en la página y de las entregas individuales de la suscripción. De algún modo es necesario combinar en la red la programación secuencial tradicional con la programación no secuencial y/o yuxtapuesta ya que el oyente elabora su propia pauta personal de secciones y programas en un tiempo o tiempos que pueden coincidir o no con la emisión en directo. Por tanto, el oyente también participa en la configuración de la oferta radiofónica y los tiempos de escucha cobran, si cabe, más relevancia.

La posibilidad de ofrecer contenidos en la red no sujetos a los horarios de emisión tradicionales, así como la capacidad de suscribir o sindicar determinados espacios incrementa la fidelización de algunos secciones o programas por parte del oyente. Durante el tiempo de estudio se ha observado que esta posibilidad es mejor aprovechada por los programas magacín de actualidad y entretenimiento, y no tanto por los programas informativos, cuyo contenido es más perecedero.
Además de la edición de la "portada informativa" para la página web -Última Hora, Noticias, Titulares, Informes Especiales, Reportajes y Coberturas en Directo-, algunas cadenas generalistas también ofrecen en la red el último boletín informativo emitido en la antena y la totalidad de los programas informativos de mañana, tarde y noche, sin realizar una fragmentación selectiva de algunas secciones del programa de mayor interés, por ejemplo, de las crónicas, de los comentarios o de las entrevistas en directo. A este aspecto, las posibilidades de escucha a demanda de contenidos informativos no deberían limitarse al programa como unidad de descarga, sino a piezas o secciones menores convenientemente indexadas para asegurar su localización. En este sentido, es clave idear una edición sonora y radiofónica completa de las diferentes unidades narrativas de la programación del día, así como cuidar la presentación destacada de la oferta a la carta de los sonidos informativos del día, los tiempos y secciones principales de los magacines y los programas temáticos. También, la organización del servicio de archivo sonoro y los sistemas de búsqueda interna en la página. El diseño y la planificación de contenidos para la red exigen, por tanto, el desarrollo de buenas herramientas de etiquetado y búsqueda, de forma que la multiplicación y diversidad de la distribución de contenidos a demanda sea más eficaz.

Otra nueva técnica a tener en cuenta desde el punto de vista de la programación es la redistribución de contenidos a través de las redes sociales que abren un abanico de nuevas posibilidades no solo para compartir contenidos, sino también para analizar el tipo de consumo que realiza el oyente en la red y ampliar la visibilidad en Internet en general. Cuestión que se volverá a abordar más adelante.

El modelo de programación de contenidos radiofónicos en la red permite compatibilizar la estrategia generalista tradicional con la atención específica y segmentada de los perfiles de públicos que conforman la audiencia variada a 
la que se dirige este tipo de radio, e interesados en determinadas noticias, temáticas de la actualidad -general y especializada- y de entretenimiento.

\subsection{Un nuevo modelo de participación y escucha}

La participación del oyente ha sido constante en la historia de la radio. Desde sus orígenes, la radio ha utilizado distintas estrategias para buscar la fidelidad de la audiencia. Los mensajes han apelado de forma continua a los oyentes, invitándoles a participar en diferentes asuntos relacionados con la actividad de la radio: desde los primeros clubes de oyentes, a través de los espacios de consulta por teléfono o por carta, en los programas de discos dedicados, en los programas de confidencias. De esta manera, se han creado lazos entre el oyente y la emisora, que comparten ideas y valores implícitos en el discurso de la radio.

La red incrementa, diversifica y amplía estas posibilidades tradicionales de participación de la audiencia de la radio, dándole mayor protagonismo y permitiendo también un diálogo o conversación entre los propios oyentes. En las temporadas analizadas, la participación del usuario en las páginas web de las cadenas generalistas estudiadas se establece a través de tres vías principales: el uso o demanda de contenidos y servicios, la aportación de nuevos contenidos generados desde la audiencia, y el establecimiento de un diálogo directo entre la emisora y su audiencia y entre las propias audiencias, a través de los blogs, comunidades de oyentes y redes sociales.

El uso o demanda de contenidos y servicios es personalizado en la red y es una modalidad clara de diálogo indirecto con la cadena. La posibilidad de suscribir y sindicar contenidos permite también que el oyente vuelva a empaquetar los contenidos en función de sus preferencias y construya una programación personal a su medida.
La producción de contenidos, de la que ya hemos hablado, es la segunda vía por la cual el oyente interactúa con su emisora. Aunque está poco explotada en los contenidos informativos de las cadenas generalistas, permite un flujo constate entre los programas y sus oyentes al participar en entrevistas digitales, encuestas, envío de fotos, etc. Para algunas coberturas especiales desde la temporada 2009/2010, se aprovecha la tecnología del microblogging de Twitter para la retransmisión y seguimiento en directo del discurso y de las opiniones que se generan entre los usuarios. La primera en utilizarlo fue Radio Nacional y lentamente comienzan a utilizarlo las demás cadenas.

La red se muestra también como un cauce novedoso de participación social, que facilita el diálogo entre aquellos perfiles de oyentes que comparten intereses comunes. Por lo tanto, la interactividad de la red fortalece la participación del oyente en el medio radiofónico y permite que sea el propio oyente el que decida las distintas formas de intervención y relación con la cadena, y con otros oyentes integrados en redes sociales. Esta nueva tendencia de participación radiofónica se produce de manera sincrónica y diacrónica con respecto de la emisión en directo.

Durante el estudio se ha constatado que la evolución en el uso de diversas modalidades de diálogo ha sido muy rápida en las últimas temporadas radiofónicas: desde el uso del correo electrónico, la constitución de foros, chats, encuestas, entrevistas electrónicas, blogs de programas y de presentadores, hasta la constitución de comunidades de oyentes que comparten intereses y la creación de espacios para la producción de contenidos, sean noticias, fotos o podcast cuyos autores son los usuarios y, por supuesto, el uso de las redes sociales. La audiencia de radio siempre ha respondido de forma activa a los requerimientos de su emisora; ahora, además, se convierte en generadora de ideas, de contenidos y de conversación. 
El siguiente paso en el que las emisoras generalistas tiene que avanzar para no perder ese diálogo con la audiencia es en potenciar sinergias de contenidos y servicios con los dispositivos móviles y los nuevos soportes digitales que empiezan a crear un nuevo mercado de consumidores de contenidos. El cambio es importante, la radio generalista tiene que salir a buscar a los oyentes en las plataformas que empiezan a imponerse para no dejar de ser un medio próximo y cercano a la audiencia, como hasta ahora.

La red, en este sentido y por ser un medio que integra diversidad de lenguajes y de formas de interactuar con la audiencia, ofrece la posibilidad de ser un terreno de desarrollo y prueba de contenidos y servicios de esas otras plataformas digitales, que con el tiempo dejarán de ser soportes para constituirse en nuevos medios.

\subsection{Comunidad de oyentes: un valor añadido para la marca radiofónica}

Por todo lo dicho, la radio adquiere una nueva dimensión o requiere un replanteamiento de conceptos al enfrentarse a la red, ahora también para identificar nuevas audiencias y desarrollar nuevas posibilidades para la marca radiofónica tradicional.

La tecnología permite diversificar los contenidos pero también impone nuevas reglas de juego en el mercado de oyentes. La necesidad de captar tanto a la audiencia que está migrando a los soportes digitales como a los oyentes potenciales, que son nativos de la red y que no conocen otra forma de consumo de medios que no sea través de una pantalla, ha llevado a las emisoras generalistas tradicionales a desarrollar con urgencia este potencial de la red. La radio generalista, que pierde lentamente oyentes en la antena, al menos en España ${ }^{7}$, empieza a ser consciente de la necesidad de idear otra formas de captar, fidelizar y medir el

7 Véase al respecto los Resúmenes Generales del Estudio General de Medios (2008, 2009, 2010 y 2011). tiempo de escucha o consumo, y de aprovechar ese intercambio con la audiencia como forma de consolidar y cuidar la marca de radio.

La red presenta mejores herramientas para conocer a los oyentes. Las estadísticas que ofrecen cada una de los contenidos de la red-en las que se mide el número de visitas, de impresiones y de envíos por correo electrónico- así como la posibilidad de descargar, sindicar, votar y compartir contenidos en redes sociales, ofrecen datos instantáneos acerca del interés de los oyentes en determinadas cuestiones. Estos datos deberían ser utilizados para incrementar la producción y el desarrollo de dichos contenidos en la red, pero también en la antena y en otros soportes, porque sin duda reflejan de forma directa los intereses de la audiencia.

La interactividad con el usuario en los contenidos ofrecidos en la red contribuye a prolongar la escucha (Pitts y Harms, 2003, 280; Ren y ChanOlmsted, 2004, 9). Aunque es importante recordar que una emisora debe dialogar siempre con sus oyentes independientemente de la tecnología que utilice como recuerda Evans $(2005,43)$. El verdadero desafío que plantea el entorno digital para una emisora de radio es mantener y fidelizar su audiencia y no tanto incrementarla (Hendy, 2000, 54). Por lo tanto, los radiodifusores tienen que aprovechar todos los recursos de la interactividad para construir y cultivar una nueva relación con su audiencia, creando comunidades de oyentes que se definen por sus intereses temáticos en torno a un tipo de programas o productos radiofónicos. La oferta de las emisoras generalistas en la red es así una oportunidad para consolidar la marca y complementar los servicios que ofrece la antena.

En la red, la radio generalista se enfrenta, además, a nuevos competidores, que como decíamos al comienzo, han incorporado en sus rutinas de producción el concepto editorial de "cierre continuo". La radio entra a competir en 
este nuevo escenario con una marca de calidad que se ha ganado en la antena y que ahora puede refrendar en la red. Su producto básico, del que parte y en el que ha forjado su marca de radio, es sonoro pero es también un conjunto de formas de hacer o rutinas profesionales para idear y producir un contenido de actualidad, entretenimiento y proximidad. A partir de estos rasgos diferenciales, que caracterizan su marca, tiene que desarrollar los contenidos propios del lenguaje y la narrativa de la red.

\section{Conclusión general}

La red está cambiando definitivamente el entorno comunicativo de los medios tradicionales y ahora también es la respuesta a un modelo de gestión de la comunicación pública que ya venía imponiéndose en los grandes grupos de comunicación a través de procesos de convergencia. Tras la convergencia, los grupos de comunicación establecen una nueva configuración y funcionamiento, primero empresarial y tecnológica, para finalmente imponer un nuevo perfil profesional y una forma de producir los contenidos (Salaverría y Negredo, 2008, 16). La red se impone como el punto de confluencia y de partida de estos nuevos planteamientos, para adquirir luego una dimensión propia y diferenciada del resto de medios tradicionales.

La radio es quizá el medio tradicional más lento en dar este paso, pero necesita realizar una apuesta estratégica convergente en la red y adaptar el prestigio y credibilidad de su marca actual al lenguaje y la narrativa de los nuevos medios. Y debe hacerlo sabiendo que su producto sonoro principal no se vende ni se consume solo -es multimedia-y que los oyentes han dejado de estar en el extremo opuesto de la comunicación para estar en el centro mismo del proceso generando, evaluando y compartiendo contenidos -es interactivo-. El centro de la comunicación radiofónica deja de estar en el soporte de difusión para pivotar sobre el contenido, la marca, la emisora -que lo idea, produce y programa-, y el oyente -que lo demanda, jerarquiza y comparte.

\section{Referencias bibliográficas}

AIMC (2008, 2009, 2010 y 2011). Estudio general de medios: Resumen general. Madrid: AIMC, en http:// www.aimc.es/

Ala-Fossi, M.; Lax, S.; O’Neill, B.; Jauert, P. y Shaw, H. (2008). "The Future of Radio is Still Digital - But Which One? Expert Perspectives and Future Scenarios for the Radio Media in 2015". En Journal of Radio \& Audio Media, vol. 15, $\mathrm{n}^{\circ}$ 1. London: Routledge, pp. 4-25.

Albarran, A. B. y Pitts, G. (2001). The Radio Broadcasting Industry. Boston: Allyn and Bacon.

Amoedo Casais, A.; Martínez-Costa, M.P.; Moreno Moreno, E. (2008). "An analysis of the communication strategies of Spanish commercial music networks on the web: los40.com, los40principales. com, cadenal00.es, europafm.es and kissfm.es". En The Radio Journal, vol. 6, $n^{\circ}$ 1. Bristol: Intellect Books, pp. 5-20.

Amoedo Casais, A.; Martínez-Costa, M.P.; Moreno Moreno, E. (2010). "La radio generalista española en la red (2006-2010): propuesta de un método de análisis". En Hacia la construcción de nuevos modelos de comunicación. Actas del II Congreso Internacional de Ciberperiodismo y Web 2.0, Universidad del País Vasco, Bilbao, 10 al 12 de noviembre de 2010, pp. 194-214.

Amoedo Casais, A.; Martínez-Costa, M. P.; y Moreno Moreno, E. (2011). "News continuity in on-air and online broadcasts on general-interest radio stations in Spain: Analyzing the three main midday news stories in the 2008-2009 and 2009-2010 seasons". En Salaverría, R. (ed.). Diversity of Journalisms. Proceedings of ECREA/CICOM Conference, Pamplona, pp. 11-23.

Asociación de la Prensa de Madrid (2008, 2009 y 2010). Informe Anual de la Profesión Periodística 2008. Madrid.

Berry, R. (2006). "Will the Ipod kill the radio star?". En Convergence, vol. 12, n 2. London: Sage, pp. 143-162. 
Canavilhas, J. (2007). Webnoticia: Propuesta de modelo periodístico para la WWW. Covilha: Labcom. En http://www. livroslabcom.ubi.pt/pdfs/canavilhas-webnoticiafinal.pdf

Calderón, A. (1972). "La dramatización radiofónica y sus conexiones con la actualidad informativa". Pamplona: Lección en el PGLA. Facultad de Ciencias de la Información. Universidad de Navarra. En Faus Belau, A. (1973). La radio: introducción al estudio de un medio desconocido. Madrid: Guadiana de Publicaciones.

Cebrián Herreros, M. (2008). La radio en internet. De la ciberradio a las redes sociales y la radio móvil. Buenos Aires: La Crujía.

Coyle, R. (2006). "Ether to 01 - Digitizing radio". En Convergence, vol. 12, n. ${ }^{\circ}$ 2. London: Sage, p. 123-127.

Díaz Noci, J. y Salaverría, R. (coords.) (2003). Manual de redacción ciberperiodística. Barcelona: Ariel.

Evans, M. (2005). "Make Internet Radio and Podcasting Part of your Arsenal". En Talkers Magazine, n. ${ }^{\circ}$ 161, September. Springfield: Focus Communications, pp. 38-39 y 43.

EVANS, C. J. y Smethers, J. S. (2001). "Streaming Into The Future. A Delphi Study of Broadcasters Attitudes Toward Cyber Radio Station". En Journal of Radio Studies, vol. 8, n. ${ }^{\circ}$ 1. Topeka: Broadcast Education Association, pp. 5-27.

Faus Belau, A. (2001). "La radio en el entorno cambiante del siglo XXI". En Martínez-Costa, M. P. (ed.). Reinventar la radio. Actas de las XV Jornadas Internacionales de Comunicación. Universidad de Navarra. Pamplona: Eunate, pp. 15-37.

Faus Belau, A. (1973). La radio: introducción al estudio de un medio desconocido. Madrid: Guadiana de Publicaciones.

Franco, G. (2008). Cómo escribir para la Web. Bases para la discusión y construcción de manuales de redacción -online. Texas: Knight Center for Journalism in the Americas, en http://knightcenter.utexas.edu/Como _ escribir_para_la_WEB.pdf
Hamula, S. R. y Wenmouth, W. J. (2003). "The Internet as a Small-Market Station Tool". En Journal of Radio Studies, vol. 10, n 2. Topeka: Broadcast Education Association, pp. 262-269.

Hendy, D. (2000). Radio in the Global Age. Cambridge: Polity Press.

Lind, R. A. y Medoff, N. J. (1999). "Radio Stations and the World Wide Web". En Journal of Radio Studies, vol. 6 , n 2. Topeka: Broadcast Education Association, pp. 203-221.

Martí, J. M. (2003). "La radiodifusión en el horizonte del tercer milenio". En López, N y Peñafiel, C. (eds.). Odisea 21: la evolución del sector audiovisual. Modos de producción cambiantes y nuevas tecnologías. Madrid: Fragua, pp. 375-386.

Martí, J. M. (2001), "Hacia un cambio de modelo: los centros de interés y las preferencias musicales". En Martínez-Costa, M. P. (ed). Reinventar la radio. Actas de las XV Jornadas Internacionales de Comunicación. Universidad de Navarra. Pamplona: Eunate, pp. 187-193.

Martínez-Costa, M. P. (2002). "El proceso de escritura de la información radiofónica". En Información radiofónica. Barcelona: Ariel. pp. 97-119.

Martínez-Costa, M. P. (2011). "La nueva organización de la producción". En Ortíz Sobrino, Miguel Angel y Lopez Vidales, Nereida (eds): Radio 3.0: una nueva radio para una nueva era. La democratización de los contenidos. Madrid: Fragua, pp. 123-140.

Martínez-Costa, M. P.; Amoedo, A.; Moreno, E. (2008). "Estrategias de comunicación de las cadenas generalistas españolas en la red: análisis comparativo de www.cadenaser.com, www.cope.es, www. ondacero.es, www.puntoradio.com y www.rne.es en las temporadas 2006-2007 y 2007-2008". Santiago de Compostela: Actas y memoria final Congreso Internacional Fundacional Asociación Española de Investigadores en Comunicación. Facultad de Ciencias de la Comunicación. 30 de enero al 1 de febrero.

Martínez-Costa, M. P.; Moreno Moreno, E.; y Amoedo Casais, A. (2009). "News Content of Spanish Talk 
Radio Networks on the Web". En New Media $\mathcal{E}$ Information: Convergentes $\&$ Divergences. Conference Proceedings, International Conference Media 2009, Atenas, Grecia.

Merayo, A. (2001). "Formación, nuevos contenidos y creatividad sonora: apuestas para un tiempo de incertidumbre tecnológica". En Martínez-Costa, M. P. (ed.). Reinventar la radio. Actas de las XV Jornadas Internacionales de la Comunicación. Universidad de Navarra. Pamplona: Eunate, pp. 281-291.

Moody, A.; Greer, J. y Linn, T. (2003). "Public Radio Station Websites \& Their Users". En Journal of Radio Studies, vol. 10, n. ${ }^{\circ}$ 2. Topeka: Broadcast Education Association, pp. 255-261.

Moreno Moreno, E.; Amoedo Casais, A.; MartínezCosta, M. P. (2008). "Radio and the Web: Communication Strategies of Spanish Radio Networks on the Web (2006-2008)". En Communication Policies and Culture in Europe, European Communication Research and Education Association, $2^{\text {nd }}$ European Communication Conference, Barcelona.

Moreno Moreno, E.; Amoedo Casais, A.; MartínezCosta, M. P. (2009). "Radio and the Web: Communication Strategies of Spanish Radio Networks on the Web (2006-2008)". En Observatory OBS, Journal 10, vol. 3, n० 3, pp. 121-137.

Moreno Moreno, E.; Amoedo Casais, A.; MartínezCosta, M. P. (2009). "News content of Spanish Talk Radio Networks on the Web", Radio Content in the Digital Age. European Communication Research and Education Association. Limassol: Cyprus University of Technology. October 14-16.

Moreno, E. (2008). "Programación radiofónica: una nueva perspectiva en el entorno digital". En Gómez, B. y Hernández, S. La periodística como disciplina universitaria: balance y perspectivas. Estudios de Periodística XIII. Actas de la IV Reunión Científica de la Sociedad Española de Periodística, 20 y 21 de abril, 2007. Pamplona: Ediciones Diario de Navarra, pp. 383-395.

Pew Research Center for the People y the Press (2008 y 2010). Audience Segments in a Changing News Environment. Key News Audiences Now Blend Online and
Traditional Sources. Pew Research Center Biennial News Consumption Survey.

Pitts, M. J. y Harms, R. (2003). "Radio Websites as a Promotional Tool". En Journal of Radio Studies, vol. 10, n. ${ }^{\circ}$ 2. Topeka: Broadcast Education Association, p. 270-282.

Priestman, C. (2004). "Narrowcasting and the dream of radio's great global conversation". En The Radio Journal, vol. 2, n 2. Topeka: Broadcast Education Association, pp. 77-88.

Priestman, C. (2002). Web radio: radio production for Internet streaming. Oxford: Focal Press.

Randle, Q. y Mordock, J. (2002). "How Radio is Adapting Weather to the Web: A Study of Weather Strategies on Local News/Talk Radio, TV, and Newspaper Home Pages". En Journal of Radio Studies, vol. 9, n. ${ }^{\circ}$ 2. Topeka: Broadcast Education Association, pp. 247-258.

Ren, W. y Chan-Olmsted, S. (2004). "Radio content on the World Wide Web: comparing streaming radio stations in the United States". En Journal of Radio Studies, vol. 11, n. ${ }^{\circ}$ 1. Topeka: Broadcast Education Association, pp. 6-25.

Ribes, X. (2003). "Internet: La Radio que se ve". En López, N. y Peñafiel, C. (eds.). Odisea 21: La evolución del sector audiovisual. Modos de producción cambiantes y nuevas tecnologías. Madrid: Fragua, pp. 89-102.

Salaverría, R. (2005). Redacción periodística en internet. Pamplona: Eunsa.

Salaverría, R. y Negredo, S. (2008). Periodismo integrado. Convergencia de medios y reorganización de redacciones. Barcelona: Editorial Sol 90 Media.

Seelig, M. (2008). "Interactivity on Traditional Media Web Sites". En Journal of Radio \& Audio Media, vol. 15, n. ${ }^{\circ}$ 2, pp. 231-248.

Tacchi, J. (2000). "The Need for Radio Theory in the Digital Age". En International Journal of Cultural Studies, vol. 3, n. ${ }^{\circ}$ 2. Londres: Sage, pp. 289-298. 
Wall, T. (2004). "The political economy of Internet music radio". EnThe Radio Journal, vol. 2, n. ${ }^{\circ}$ 1. Bristol: Intellect Books, pp. 27-44.
Waghorn, R. (2005). "El modelo ingles en DAB y DBT". En Peinado M., Rodríguez, M. D. y Fernández, M. A., La radio y la televisión en la Europa digital. Seminario Internacional Complutense. Madrid: Facultad de Ciencias de la Información, pp. 35-41. 